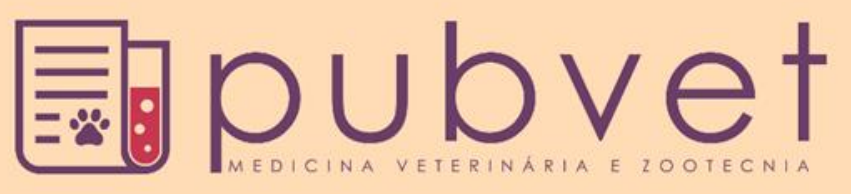

ISSN $1982-1263$

https://doi.org/10.31533/pubvet.v13n4a306.1-7

\title{
Hidratação enteral em fluxo contínuo no tratamento da compactação de cólon maior em um equino: Relato de caso
}

\author{
Elson Nery da Silva ${ }^{1 *} \bullet$, Marta Eloy Nunes da $\operatorname{Cunha}^{1}{ }^{\bullet}$, Domingos Cachineiro Rodrigues Dias ${ }^{\circ}{ }^{\bullet}$, Daniel

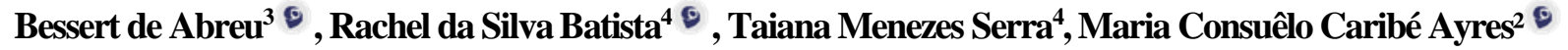 \\ ${ }^{I}$ Médico(a) Veterinário(a) Residente em Clínica de Ruminantes e Equídeos, Universidade Federal da Bahia, Salvador, Brasil \\ ${ }^{2} \operatorname{Prof}^{\circ}(a)$ do Departamento de Anatomia, Patologia e Clínicas, Universidade Federal da Bahia, Salvador, Bahia, Brasil. \\ ${ }^{3}$ Médico veterinário, Coordenador do Departamento Técnico da Empresa Matsuda-Vitória da Conquista Bahia, Brasil \\ ${ }^{4}$ Estagiárias do curso de medicina veterinária, Universidade Federal da Bahia, Salvador, Brasil \\ *Autor para correspondência, E-mail: elsonneryd@msn.com
}

Resumo. A síndrome cólica equina é a principal responsável pelo óbito de cavalos em todo mundo. Ela é caracterizada basicamente por dor abdominal aguda, sendo que uma das suas etiologias mais comuns é a compactação de cólon maior. Em novembro de 2017, um equino macho, castrado, de pelagem alazã, sem raça definida, com 7 anos de idade, foi encaminhado para o Setor de Equídeos do Centro de Desenvolvimento da Pecuária (CDP) da Universidade Federal da Bahia (UFBA) com queixa principal de desconforto abdominal e ausência de defecação há cerca de um dia, histórico de estar sendo mantido em pasto inadequado e submetido à restrição hídrica. No atendimento clínico foi determinado quadro de compactação de cólon maior. Inicialmente foi realizado tratamento com dipirona/escopolamina e hidratação intravenosa com solução de ringer lactato. Porém, após algumas horas, optou-se pela hidratação enteral em fluxo contínuo (HET) como terapia hidroeletrolítica num regime de $15 \mathrm{ml} / \mathrm{kg} / \mathrm{h}$ utilizando solução hipotônica. Após seis dias houve remissão dos sinais de desconforto abdominal, retorno da motilidade intestinal e da defecação e alteração do aspecto das fezes de ressecadas a amolecidas. A hidratação intravenosa com solução de ringer lactato, apesar de ser a mais utilizada, apresenta algumas desvantagens como seu alto custo, sendo a HET com solução poliônica hipotônica eficiente e muito mais econômica no tratamento da compactação no cólon maior em equinos.

Palavras-chave: cavalo, cólica, compactação, hidratação enteral

\section{Continuous flow enteral hydration in treatment of larger bowel compaction in an equine: Case report}

\begin{abstract}
Equine colic syndrome is the main cause of horse death worldwide. It is characterized primarily by acute abdominal pain, one of its most common etiologies being compression of the colon. In November 2017, a seven year old male, castrated, brownskinned equine was sent to the Equine Sector of the Centro de Desenvolvimento da Pacuária (CDP) of the Universidade Federal da Bahia (UFBA) with main complaint of abdominal discomfort and absence of defecation for about a day, a history of being kept in inappropriate pasture and submitted to water restriction. In the clinical care, a larger colon compaction was determined. Initially, treatment with dipyrone/scopolamine and intravenous hydration with lactated ringer solution was performed. However, after a few hours, continuous-flow enteral hydration was chosen as hydroelectrolytic therapy at a rate of $15 \mathrm{ml} / \mathrm{kg} / \mathrm{h}$ using hypotonic solution. After six days there was remission of signs of abdominal discomfort, return of intestinal motility and defecation and alteration of the appearance of dry stools to softened stools. Intravenous hydration with lactated ringer
\end{abstract}


solution, despite being the most used, presents some disadvantages as its high cost, with continuous-flow enteral hydration with polyotonic solution hypotonic efficient and much more economical in the treatment of compaction in the greater colon in equines.

Key words: horse, colic, compression, enteral hydration

\title{
Hidratación enteral en flujo continuo en el tratamiento de la compactación de colon mayor en equino: Reporte de un caso
}

\begin{abstract}
Resumen. El síndrome cólico equina es el principal responsable de la muerte de caballos en todo el mundo. Se caracteriza básicamente por dolor abdominal agudo, siendo que una de sus etiologías más comunes es la compactación de colon mayor. En noviembre de 2017, un equino macho, castrado, de pelaje alazán, sin raza definida, con 7 años, fue encaminado al sector de équidos del Centro de Desenvolvimento da Pecuária (CDP) de la Universidade Federal de Bahia (UFBA) con la queja principal de incomodidad abdominal y ausencia de defecación hace cerca de un día, histórico de estar siendo mantenido en pasto inadecuado y sometido a la restricción hídrica. En la consulta clínica fue determinado cuadro de compactación de colon mayor. Inicialmente se realizó tratamiento con dipirona/escopolamina e hidratación intravenosa con solución de ringer lactato. Sin embargo, después de algunas horas, se optó por la hidratación enteral en flujo continuo (HET) como terapia hidroelectrolítica en un régimen de $15 \mathrm{ml} / \mathrm{kg} / \mathrm{h}$ utilizando solución hipotónica. Después de seis días hubo remisión de los signos de incomodidad abdominal, retorno de la motilidad intestinal y de la defecación y alteración del aspecto de las heces de resecadas a ablandadas. La hidratación intravenosa con solución de ringer lactato, a pesar de ser la más utilizada, presenta algunas desventajas como su alto costo, siendo la HET con solución poliónica hipotónica eficiente y mucho más económica en el tratamiento de la compactación en el colon mayor en equinos.
\end{abstract}

Palabras clave: caballo, cólico, compactación, hidratación enteral

\section{Introdução}

Os cavalos podem ser acometidos por síndrome cólica, que é um termo utilizado para descrever a dor de origem visceral em equinos. Na maioria dos casos, é ocasionada por distúrbios gastrointestinais. Trata-se de uma das principais enfermidades que acometem os equinos na rotina da clínica equina (Laranjeira \& Almeida, 2009). Esta enfermidade é responsável por perdas econômicas devido a gastos com o tratamento, o período de afastamento dos animais de suas atividades e ao risco de óbito do animal (Reed et al., 2009; Thomassian, 2005).

De forma geral na medicina equina, a cólica é uma síndrome caracterizada principalmente por dor visceral aguda dependendo do grau de acometimento das alças intestinais, tais sinais são geralmente decorrentes de disfunção do trato gastrintestinal, entretanto, algumas doenças com origem em outros órgãos não gastrintestinais podem causar sinais clínicos de dor visceral. Dentre as variadas causas de síndrome cólica, estão às compactações intestinais, acúmulos de ingesta desidratadas, que ocorrem em qualquer segmento do cólon maior, levando a obstruções do trânsito intestinal. Estas alterações também podem ocorrer no estômago, intestino delgado, ceco, cólon transverso e colón menor. A flexura pélvica é o segmento com maior ocorrência de compactação, decorrente do estreitamento do lúmen nessa região (Reed et al., 2009; Thomassian, 2005). O tratamento básico consiste no manejo da dor associado à hidratação intravenosa, ocasionalmente associada com a hidratação enteral. Porém, nos últimos anos têm se demonstrado a grande importância da hidratação enteral como modalidade eficiente e econômica de terapia hidroeletrolítica em equinos (Ribeiro Filho et al., 2009), principalmente como tratamento de eleição para as compactações simples de cólon maior (Lopes, 2002). Cavalos com compactação de intestino grosso frequentemente se beneficiam da administração de fluido enteral (Mair, 2002; Southwood, 2006).

Com este estudo objetivou-se relatar um caso clínico, de um equino com Síndrome Cólica Equina por compactação do cólon maior, onde o tratamento utilizando Hidratação Enteral (HET) em Fluxo 
Contínuo obteve sucesso e cura do paciente, na Clínica de Equídeos do Centro de Desenvolvimento da Pecuária (CDP) da Universidade Federal da Bahia (UFBA), situado no município de Santo Amaro - BA.

\section{Relato do caso}

Em novembro de 2017, deu entrada no Centro de Desenvolvimento da Pecuária, da Escola de Medicina Veterinária e Zootecnia da Universidade Federal da Bahia, um equino macho castrado, 7 anos de idade, $360 \mathrm{~kg}$, pelagem alazã, sem raça definida, com quadro de síndrome cólica.

$\mathrm{Na}$ anamnese, o proprietário relata o animal não defecar a 24 horas e inquietação na baia. $\mathrm{O}$ animal era mantido semi estabulado com oferta de capim picado e concentrado, $2 \mathrm{~kg}$, duas vezes ao dia. Ainda, de acordo com o proprietário, o volumoso fornecido não era de boa qualidade e o animal estava sendo submetido à restrição hídrica por problemas de manejo na propriedade. Sem histórico de vacinação e vermifugação recentes.

Ao exame físico geral, o paciente apresentou-se semi-apático com frequência cardíaca (54 bpm) frequência respiratória $(12 \mathrm{mrm})$, temperatura de $38.9^{\circ} \mathrm{C}$, tempo de preenchimento capilar de (3 seg.), mucosa oral seca com ocular levemente amarelada, retração do globo ocular (enoftalmia), hipomotilidade à auscultação abdominal, com base nos parâmetros físicos o grau de desidratação foi estimado em $8 \%$. A sondagem nasogástrica foi feita com uma sonda de silicone com $15 \mathrm{~mm}$ de espessura a fim de realizar esvaziamento e lavagem gástrica por sifonagem, não sendo observado refluxo espontâneo. Após palpação retal, detectou-se compactação de cólon maior com um acúmulo maior que $30 \mathrm{~cm}$ de comprimento. $\mathrm{O}$ conjunto de achados clínicos e informações obtidas na anamnese levaram a um diagnóstico de compactação de cólon maior, e pela manifestação branda do caso optou-se pelo tratamento clínico. Seu hemograma apresentou hematócrito de 32\%, VGM de 51,6fL, CHGM de 33,1\%, fibrinogênio de $600 \mathrm{mg}$ dl-1 e 6.250 leucócitos/ $\mu \mathrm{L}$. Avaliação clínica e hematológica foi realizada durante todo período de acometimento da enfermidade estão expostos na Tabela 1.

Tabela 1. Valores hematológicos durante o período de internamento

\begin{tabular}{|c|c|c|c|c|c|c|}
\hline \multirow{2}{*}{ Parâmetros } & \multicolumn{5}{|c|}{ Valores Encontrados } & \multirow{2}{*}{$\begin{array}{l}\text { Valores de Referência } \\
\text { Cowell \& Tyler (2009) }\end{array}$} \\
\hline & $1^{\circ}$ & $2^{\circ}$ & $3^{\circ}$ & $4^{\circ}$ & $5^{\circ}$ & \\
\hline Hematócrito (\%) & 32 & 30 & 30 & 24 & 28 & $32,0-48,0$ \\
\hline Hemácias $\left(\mathrm{x} 10^{6} / \mu \mathrm{L}\right)$ & 6,2 & 5,63 & 5.75 & 4,7 & 5,75 & $6,4-12,0$ \\
\hline Hemoglobina (g/dL) & 10,6 & 10 & 10 & 8 & 9,3 & $10,0-18,0$ \\
\hline VGM (fL) & 51,6 & 53,2 & 52 & 51 & 48,6 & $34,0-58,0$ \\
\hline CHGM (\%) & 33,1 & 33,3 & 33 & 33,3 & 33,2 & $31,0-37,0$ \\
\hline PPT (g/dL) & 7 & 7 & 7 & 6,8 & 8 & $6,0-8,5$ \\
\hline Fibrinogênio & 600 & 600 & 400 & 800 & 1.200 & $100-500$ \\
\hline Leucócitos $(/ \mu \mathrm{l})$ & 6.250 & 7.350 & 7.300 & 9600 & 11.550 & $6.000-12.000$ \\
\hline Bastonetes & 0 & 662 & 438 & 0 & 402 & $0-100$ \\
\hline Segmentados & 3.750 & 4.925 & 5.037 & 6.912 & 8.085 & $3.000-6.000$ \\
\hline Eosinófilos & 126 & 0 & 146 & 192 & 578 & $0-800$ \\
\hline Basófilo & 0 & 0 & 73 & 96 & 0 & $0-300$ \\
\hline Linfócitos & 1.750 & 1.397 & 11.314 & 1728 & 2.195 & $1.500-5.000$ \\
\hline Monócitos & 626 & 368 & 292 & 672 & 231 & $0-1000$ \\
\hline
\end{tabular}

Como tratamento, foi realizada a hidratação intravenosa em um volume total de 17 litros com ringer lactato, aplicação de $20 \mathrm{~mL}$ de dipirona sódica $\left(25 \mathrm{mg} / \mathrm{kg}\right.$ ) com escopolamina (Buscofin ${ }^{\circledR}$ ) por via intravenosa e adotou-se a hidratação enteral (Figura 1) em fluxo contínuo para manutenção com solução hipotônica composta de $4 \mathrm{~g} / 1$ de cloreto de sódio $(\mathrm{NaCl}), 0,5 \mathrm{~g} / \mathrm{l}$ de cloreto de potássio $(\mathrm{KCl}), 1 \mathrm{~g} / \mathrm{l}$ de Acetato de Cálcio $\left(\mathrm{C}_{2} \mathrm{H}_{3} \mathrm{O}_{2}\right), 0,2 \mathrm{~g} / \mathrm{l}$ de Cloreto de Magnésio $(\mathrm{MgCl} 2)$ e $5 \mathrm{~g} / \mathrm{l}$ de maltodextrina, numa taxa de $15 \mathrm{ml} / \mathrm{kg} / \mathrm{h}$ num total de cerca de 60 litros por dia. Após seis dias de terapia hídrica, houve a resolução da síndrome cólica, com retorno da defecação espontânea e normal, porem se manteve a hidratação enteral por mais dois dias sendo retirada gradualmente. Considerou-se curado o animal que não 
apresentava mais qualquer sinal de compactação do cólon maior, isto é, ausência de dor, pulso normal, alimentação normal, hidratado e com fezes normais espontâneas (Figura 4). O exame físico foi realizado conforme descrito por Speirs (1999) e a palpação retal seguindo orientações de Alves et al. (2008).

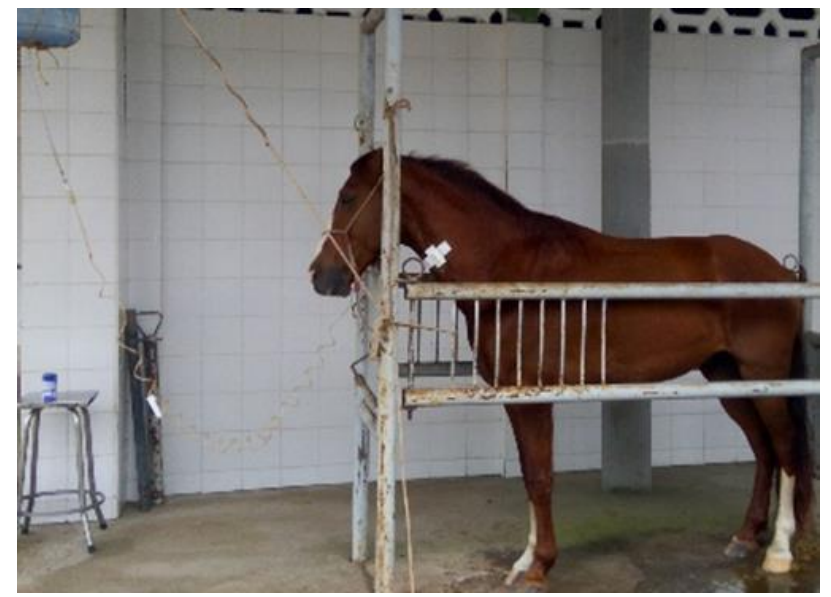

Figura 1. Cavalo recebendo hidratação enteral por sonda de pequeno calibre

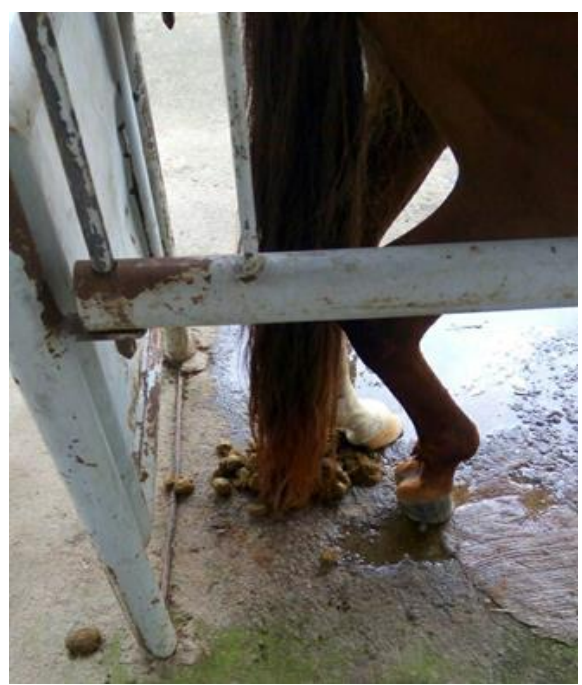

Figura 3. Fezes ressecadas

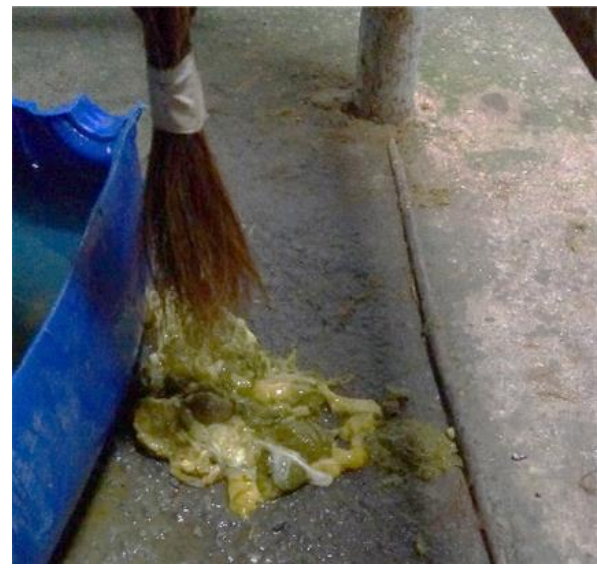

Figura 2. Fezes com presença de muco

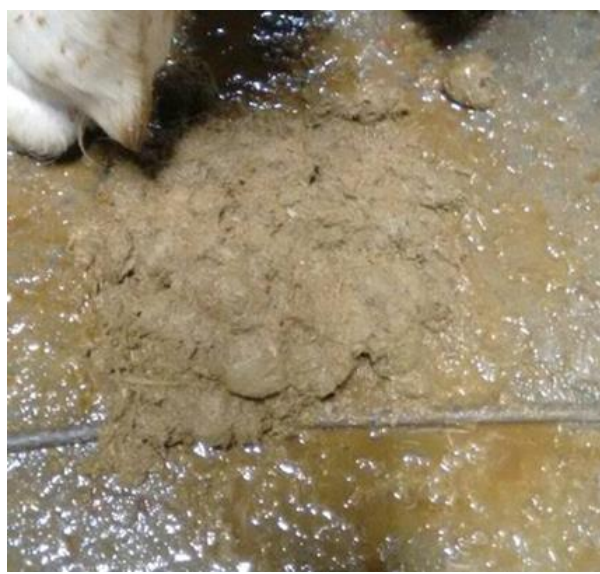

Figura 4. Fezes hiperidratadas após hidratação enteral

\section{Discussão}

Em clínicas de referência nos Estados Unidos da América (EUA), as compactações do cólon maior constituem cerca de $13 \%$ dos casos de cólica atendidos e a mortalidade nesses casos atinge os $20 \%$ (Dabareiner \& White, 1997). No caso exposto, o capim picado era de variadas espécies sendo à base da alimentação volumosa. Este também pode ser proveniente de margens de rodovias, hábito comum em todo território nacional (Cação, 2017). Acredita-se que o fornecimento de capim muito fibroso é uma das maiores causas de compactação do intestino grosso em cavalos (Lopes et al., 1998). Mesmo assim, alimentar equídeos com capim picado é uma prática comum nos estados do Nordeste brasileiro. Pessoa et al. (2012) verificaram que (37,1\% dos casos de cólica) como sendo provavelmente, reflexo do tipo de alimentação oferecida aos animais, sendo que (48,6\% do total de animais com cólica por compactação) estiveram associados ao consumo de capim elefante picado. Animais que consomem algum tipo de capim triturado são mais propícios à síndrome cólica (Dias et al., 2012). O capim elefante picado é bem difundido no Brasil devido a sua alta produtividade e boa adaptabilidade (Diehl et al., 2014; Vale \& Azevedo, 2013). A qualidade do volumoso, a disponibilidade de água, problemas de dentição, falta de exercício e mudanças recentes no manejo (extensivo para intensivo) podem ser causas de cólica por compactação em equinos (Dias et al., 2012; Keller, 1985; Schumacher \& Mair, 2002; Thomassian, 2005). 
O equino não tinha uma raça definida. Isso pode ser um fator ao desenvolvimento da cólica visto que Mariano et al. (2011) revisando estudo retrospectivo relacionado ao desenvolvimento de cólica relata haver predomínio de animais da raça BH (Brasileira de Hipismo); mas tal fato pode ser associado à sua localização geográfica e dos centros de referência utilizados. Mariano et al. (2011) relatam que vários estudos sugeriram que animais de outras raças como Árabe, PSI (Puro-sangue Inglês) também são mais susceptíveis que outras raças, porém está afirmação leva muito em conta localização geográfica do estudo.

Na palpação retal observou-se uma grande quantidade de muco e poucas sibalas endurecidas (Figura 2), concordando com as descrições de Thomassian (2005) que atribui o ressecamento das fezes a uma baixa ingestão de água, desidratação e/ou diminuição no trânsito intestinal, além da formação de uma película de cobertura (muco) que é produzida para proteger a mucosa intestinal e facilitar o deslizamento da ingesta no trânsito parado ou diminuído. Geralmente, animais com compactação, a produção fecal está reduzida (Reed et al., 2009) e as fezes geralmente apresentam-se duras, ressecadas (Figura 3), pequenas e cobertas de muco (Reed et al., 2009) ou amolecidas e em quantidades reduzidas (Ribeiro Filho \& Alves, 2002). A presença de fezes ressecadas evidencia-se pela diminuição do grau de umidade e quando recoberta por muco significa uma lentidão no trânsito intestinal. Ferreira et al. (2009) descrevem que a maioria dos animais, com compactação do colón maior, apresentaram fezes pastosas e fibrosas ou não apresentam fezes no momento do atendimento clínico. A alteração do conteúdo fecal sempre deve ser avaliada no exame clínico, pois qualquer alteração indica disfunção intestinal, por absorção ou secreção exacerbada, podendo funcionar como indicador de distúrbio gastrintestinal (Laranjeira \& Almeida, 2009).

As vias de administração de fluídos comumente utilizadas em equinos são a intravenosa e a nasogástrica. A hidratação enteral (HET) consiste na administração de soluções eletrolíticas através de sonda nasogástrica comumente utilizada para lavagem estomacal. As vantagens da HET são incontestáveis, entretanto o método tradicional usado em equinos, que é através de uma sonda nasogástrica de grosso calibre, tem algumas limitações: a passagem da sonda é trabalhosa, podendo causar traumas e sangramentos na cavidade nasal e ao esôfago; o animal não deve ser deixado com a sonda durante muito tempo, é preciso administrar fluído várias vezes ao dia para que grandes volumes de fluidos possam ser administrados, expondo-o várias vezes ao estresse e desconforto se as quantidades máximas não forem respeitadas (Ribeiro Filho \& Alves, 2002). Uma alternativa para administração de fluidos é a via nasogástrica, com o emprego de sonda de pequeno calibre (Avanza et al., 2009). O uso desta técnica, empregada com sucesso em equinos, permite que os animais sejam mantidos em baias sem contenção enquanto a hidratação é administrada continuamente sendo seguro permitir o acesso a alimentos (Avanza et al., 2009). A hidratação enteral usada para manutenção da hidratação apresentou baixo custo, menor que a solução utilizada por via intravenosa para repor a volemia.

Quando internado, o paciente apresentava diminuição da elasticidade da pele, enoftalmia, TPC elevado, mucosas ressecadas e fezes secas. Estes sinais indicavam desidratação (Belli et al., 2008) que é frequentemente observada nos casos de compactação do cólon maior (Dibartola, 2000). Os sinais de desidratação não eram mais observados a partir do segundo dia do tratamento, indicando que a hidratação enteral foi eficiente em reestabelecer e manter a hidratação. Durante a fase de hidratação, foi constatada diminuição nos valores do hematócrito, demonstrando a eficácia da hidratação enteral (HET) na expansão do volume plasmático, corroborando com os achados de Lopes (2002) e Alves et al. (2005). Contudo pós a administração da HET, não foi observada também redução da PPT nos dois primeiros dias apenas a partir do terceiro dia.

\section{Conclusões}

A hidratação enteral administrada por sonda nasogástrica de fino calibre de forma contínua no volume de $15 \mathrm{~mL} \mathrm{kg-1} 12 \mathrm{~h}-1$ perfazendo um volume diário total de 60 litros, promoveu melhora no quadro clínico do animal. A HET associada ao controle da dor mostrou-se uma alternativa eficaz, segura e muito mais econômica para o tratamento da compactação de cólon maior que não possuem indicação cirúrgica. 


\section{Referências bibliográficas.}

Alves, G. E. S., Botteon, P. T. L., Oliveira, H. P. \& Cassou, F. (2008). Exame transretal do equino com cólica. Cadernos Técnicos de Veterinária e Zootecnia, 5847-106.

Alves, G. E. S., Ribeiro Filho, J. D., Oliveira, H. P. \& Abreu, J. M. G. (2005). Tratamento da compactação experimental do cólon maior em equinos: resultados de laboratório e exames bioquímicos. Arquivo Brasileiro de Medecina Veterinária e Zootecnia, 57281-287.

Avanza, M. F. B., Ribeiro Filho, D., Lopes, M. A. F., Ignácio, F. S., Carvalho, T. A. \& Guimarães, J. D. (2009). Hidratação enteral em equinos-solução eletrolítica associada ou não à glicose, à maltodextrina e ao sulfato de magnésio: resultados de laboratório. Ciência Rural, 39(4):1126-1133.

Belli, C. B., Michima, L. E. d. S., Latorre, S. M. \& Fernandes, W. R. (2008). Solução concentrada de albumina equina na fluidoterapia em equinos com desidratação leve a moderada. Arquivos Brasileiros de Medicina Veterinária e Zootecnia, 60(1):30-35.

Cação, M. M. F. (2017). "Capim BR”. Pesquisa \& Tecnologia, 14(1):1-6.

Cowell, R. L. \& Tyler, R. D. (2009). Diagnostic cytology and hematology of the horse E-Book. Amsterdam, Holanda: Elsevier Health Sciences.

Dabareiner, R. M. \& White, N. A. (1997). Diseases and surgery of the cecum. Veterinary Clinics of North America: Equine Practice, 13(2):303-315.

Dias, R. V. C., Souza, M. V. \& Ribeiro Filho, J. D. (2012). Potenciais fatores de risco da síndrome cólica em equinos. Revista CFMV, 18(56):35-42.

Dibartola, S. P. (2000). Introduction to fluid therapy. In S. P. DiBartola (Ed.), Fluid therapy in small animal practice (pp. 265-280). Philadelphia, USA: W.B. Sauders.

Diehl, M. S., Olivo, C. J., Agnolin, C. A., Azevedo Junior, R. L., Bratz, V. F. \& Santos, J. C. (2014). Massa de forragem e valor nutritivo de capim elefante, azevém e espécies de crescimento espontâneo consorciadas com amendoim forrageiro ou trevo vermelho. Ciência Rural, 44(10):1845-1852.

Ferreira, C., Palhares, M. S., Melo, U. P., Gheller, V. A. \& Braga, C. E. (2009). Cólicas por compactação em equinos: etiopatogenia, diagnóstico e tratamento. Acta Veterinaria Brasilica, 3(3):117-126.

Keller, S. D. (1985). Diseases of the equine small colon. The Compendium on Continuing Education for the Practicing Veterinarian, 7113-120.

Laranjeira, P. V. E. H. \& Almeida, F. Q. (2009). Síndrome cólica em equinos: ocorrência e fatores de risco. Revista de Ciências da Vida, 28(1):64-78.

Lima, R. A. S., Shirota, R. \& Barros, G. S. C. (2006). Estudo do complexo do agronegócio cavalo. Escola Superior de Agricultura Luiz de Queiroz, ESALQ.: Confederação da Agricultura e Pecuária do Brasil.

Lopes, M. A. F. (2002). Physiological aspects, indications and contraindications of enteral fluid therapy. Equine Veterinary Education, 14(5):257-262.

Lopes, M. A. F., Moura, G. S., Junqueira, L. A. C., Lima, L. R., Pinto, J. O., Ribeiro Filho, J. D. \& Assis, C. B. (1998). Tratamento de compactação do cólon maior em equídeos com fluidoterapia enteral. Ciência Rural, 28(3):417-422.

Mair, T. S. (2002). Contributions to an evidence-based medicine approach to colic surgery. Equine Veterinary Journal, 34(5):428-429.

Mariano, R. S. G., Pacheco, A. M., Hamzé, A. L., Abilio, A. F. \& Avanza, M. F. B. (2011). Síndrome Cólica Equina - Revisão de Literatura. Revista Científica Eletrônica de Medicina Veterinária, 9116.

Pessoa, A. F. A., Miranda Neto, E. G., Ricardo, C., Pessoa, S. V. D. M., Simões, S. S. A. \& Riet-Correa, F. (2012). Abdômen agudo em equídeos no semiárido da região Nordeste do Brasill. Pesquisa Veterinária Brasileira, 32(6):503-509.

Reed, S. M., Bayly, W. M. \& Sellon, D. C. (2009). Equine internal medicine: Elsevier Health Sciences.

Ribeiro Filho, J. D. \& Alves, G. E. S. (2002). Compactação no intestino grosso de eqüinos: estudo comparativo de três protocolos de indução. Arquivo Brasileiro de Medicina Veterinária e Zootecnia, 54370-375. 
Ribeiro Filho, J. D., Gomes, C. L. N., Fonseca, B. P. A. \& Pinto, J. O. (2009). Hidratação enteral em ruminantes e equídeos. Eficiência com menor custo. Revista do Conselho Federal de Medicina Veterinária, 15(48):63-67.

Schumacher, J. \& Mair, T. S. (2002). Small colon obstructions in the mature horse. Equine Veterinary Education, 14(1):19-28.

Southwood, L. L. (2006). Acute abdomen. Clinical Techniques in Equine Practice, 5(2):112-126.

Speirs, V. C. (1999). Exame clínico de eqüinos. Porto Alegre, Rio Grande do Sul, Brasil: Artmed.

Thomassian, A. (2005). Enfermidades dos cavalos. 4 ed. Sao Paulo: Livraria Varela, 2005. cap 2, p $42-$ 43. Sao Paulo, Brasil: Livraria Varela.

Vale, M. B. \& Azevedo, P. V. (2013). Avaliação da produtividade e qualidade do capim elefante e do sorgo irrigados com água do lençol freático e do rejeito do dessalinizador. Holos, 3181-195.

Recebido: 21 janeiro, 2019.

Aprovado: 1 de março, 2019

Publicado: 15 de abril, 2019.

Licenciamento: Este artigo é publicado na modalidade Acesso Aberto sob a licença Creative Commons Atribuição 4.0 (CC-BY 4.0), a qual permite uso irrestrito, distribuição, reprodução em qualquer meio, desde que o autor e a fonte sejam devidamente creditados 